\title{
The vertical metallicity gradients of mono-age stellar populations in the Milky Way thin disk
}

\author{
Ioana Ciucă ${ }^{1}$, Daisuke Kawata ${ }^{1}, J$ ane $\operatorname{Lin}^{2}$, Luca Casagrande ${ }^{2}$, George \\ Seabroke $^{1}$ and Mark Cropper ${ }^{1}$ \\ ${ }^{1}$ Mullard Space Science Laboratory, University College London, Holmbury St. Mary, Dorking, \\ Surrey, RH5 6NT, UK \\ email: ioana.ciuca.16@ucl.ac.uk \\ ${ }^{2}$ Research School of Astronomy \& Astrophysics, Australian National University, Canberra \\ ACT 2611, Australia
}

\begin{abstract}
We investigate the vertical metallicity gradients of five mono-age stellar populations between 0 and 11 Gyr for a sample of 18435 dwarf stars selected from the cross-matched TychoGaia Astrometric Solution (TGAS) and RAdial Velocity Experiment (RAVE) Data Release 5. We find a correlation between the vertical metallicity gradients and age, with no vertical metallicity gradient in the youngest population and an increasingly steeper negative vertical metallicity gradient for the older stellar populations. We also find that the intrinsic dispersion in metallicity increases steadily with age. Our results are consistent with a scenario that thin disk stars formed from a flaring thin star-forming disk.
\end{abstract}

Keywords. Galaxy: disk - mono-age stellar populations - vertical metallicity gradient

\section{Introduction}

The ultimate goal of Galactic archaeology in the Milky Way is to reconstruct the Galaxy's formation and evolution history. For this purpose, one of the most cogent approaches is to map the chemo-dynamical structure of the Galaxy in chronological order. The existence of a vertical metallicity gradient in a mono-age stellar population of the current Galactic disk provides strong constraints on the formation and evolution history of the Galactic disk (e.g. Kawata et al. 2017; Schönrich et al. 2017). Also, measuring the change of the vertical metallicity gradient with age gives stronger constraints. Recently, Gaia Collaboration et al. $(2016 \mathrm{a}, \mathrm{b})$ released their first Gaia data, including the Tycho-Gaia Astrometric Solution dataset (TGAS; Michalik et al. 2015), which provides accurate astrometry for more than 2 million bright stars. At the same time, the RAVE survey team made their fifth data release (DR5; Kunder et al. 2017), consisting of carefully calibrated metallicity and stellar parameters of more than 200000 stars that are also present in the TGAS which provides accurate parallax, i.e. distance information. Using the combined data set of TGAS and RAVE, we measure the isochrone ages for the dwarf stars and perform robust linear regression based on the Bayesian hierarchical model of Kelly (2007) to obtain a new measurement of the vertical metallicity gradients as a function of age for Solar neighbourhood stars.

\section{Results and conclusion}

All our mono-age stellar populations show a negative vertical metallicity gradient as seen in the left panel of Fig. 1. There appears to be a steady increase in steepness 

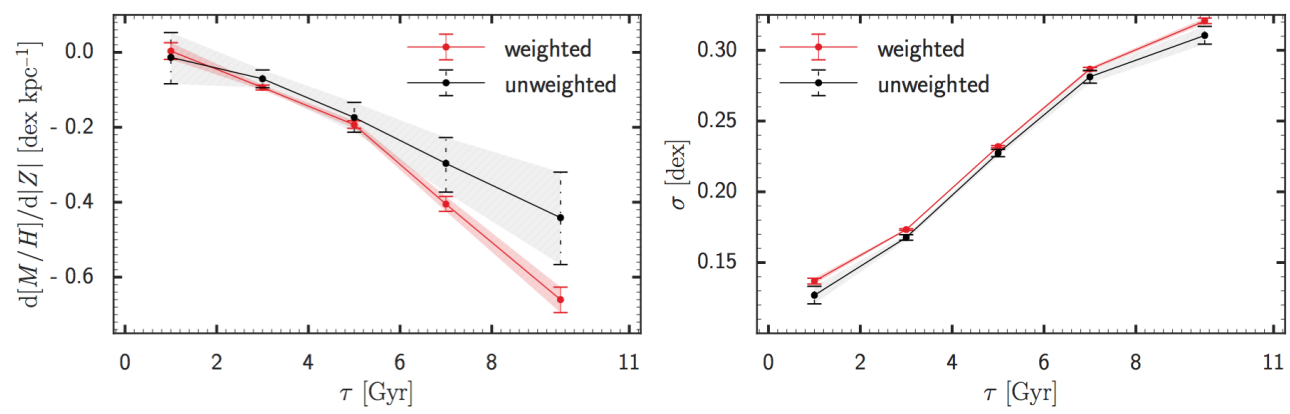

Figure 1. The best-fit estimates and their uncertainties for the slope (upper panel) and dispersion (lower panel) as a function of age, in both the stellar population bias weighted (light red) and unweighted (black) cases. The shaded area gives the uncertainty space.

of the negative slope with increasing age. The youngest population of stars with ages between 0 and 2 Gyr show an almost flat vertical metallicity gradient, $\mathrm{d}[M / H] / \mathrm{d}|Z|$

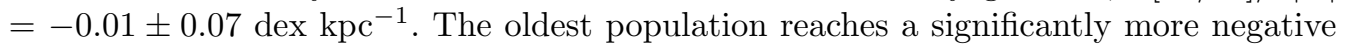
value, at $\mathrm{d}[M / H] / \mathrm{d}|Z|=-0.44 \pm 0.12 \mathrm{dex} \mathrm{kpc}^{-1}$ in the case of the stellar population bias unweighted case (see Casagrande et al. 2016; Ciucă et al. 2017 for a more in depth discussion on selection bias). This trend is also observed in Xiang et al. (2015) who used turn-off stars in the LAMOST data. The intrinsic scatter increases steadily with age from 0.13 dex for the youngest population to 0.31 dex for the oldest population as shown in the right panel of Fig. 1. The larger intrinsic dispersion of the metallicity distribution of the older stellar populations can be explained by a radial mixing mechanism which brings more metal-rich (poor) stars from the inner (outer) region of the Galactic disk for older stars. The steady increase of the dispersion indicates that radial mixing has not reached saturation, which is expected to happen if the radial mixing processes mix stars born at different radii quickly.

Our results support a model of Kawata et al. (2017) where they consider that each mono-age stellar population formed from a flaring star-forming disk. Kawata et al. (2017) demonstrate that if this flaring thin disk has a negative radial metallicity gradient when the mono-age stellar populations formed, then radial mixing processes could bring the metal poor stars formed in the flared outer disk to high vertical height in the inner disk, which leads to a negative vertical metallicity gradient. If this process is slow enough, we expect to see the older population having a steeper negative vertical metallicity gradient.

\section{References}

Casagrande L., et al., 2016, MNRAS, 455, 987.

Ciucă I., Kawata D., Lin J., Casagrande L., Seabroke G., Cropper M., 2017, preprint, (arxiv:1706.05005).

Gaia Collaboration et al., 2016a, A\&A, 595, A1.

Gaia Collaboration et al., 2016b, A\&A, 595, A2.

Kawata D., Grand R. J. J., Gibson B. K., Casagrande L., Hunt J. A. S., Brook C. B., 2017, MNRAS, 464, 702.

Kelly B. C., 2007, ApJ, 665, 1489.

Kunder A., et al., 2017, AJ, 153, 75.

Michalik D., Lindegren L., Hobbs D., 2015, A\&A, 574, A115.

Schönrich R., McMillan P. J., 2017, MNRAS, 467, 1154.

Xiang M.-S., et al.,, 2015, Research in Astronomy and Astrophysics, 15, 1209. 